hair cells. As noted by Chen et al ${ }^{1}$, selective inactivation of $\mathrm{p} 27^{\mathrm{Kip} 1}$ in support cells would be anticipated to allow proliferation, although it is unclear whether the developmental switch responsible for imposing Ink $4 d$ expression to facilitate conversion to a hair cell could be re-instated under such conditions.

Through analysis of mice lacking one or more of the seven CKIs in mammals, considerable progress has been made in understanding the mechanisms used by cells to maintain the post-mitotic state during development ${ }^{3-5}$. Numerous examples of developmental collaboration between CKIs have been found ${ }^{10,14,15}$. Although Kip1 and Ink4d provide redundant functions in the developing brain of mice ${ }^{10}$, Chen et al. show that postnatal sensory hair cells of the Organ of Corti depend only on Ink4d to maintain the post-mitotic state. Understanding the nature of the specific functions of these proteins in maintaining neural circuitry may elucidate mechanisms of sensory loss. However, reconstitution of these circuits will be a challenge. Even if a formula for deriving a hair cell from a postnatal support cell is ultimately developed, the rewiring of that hair cell into a preexisting neural network will probably be a daunting task. It may prove that sensory receptors and neural circuits can only interact if they are built together, from the ground up.

Bridget Baumgartner is in the Program in Cellular and Molecular Biology, One Baylor Plaza, Baylor College of Medicine, Houston, TX 777030, USA. $J$. Wade Harper is in the Program in Cellular and Molecular Biology, Department of Biochemistry and Molecular Biology and Department of
Molecular Physiology and Biophysics, One Baylor Plaza, Baylor College of Medicine, Houston, TX 777030, USA.

e-mail: jharper@bcm.tmc.edu

1. Chen et al. Nature Cell Biol. 5, 422-426 (2003).

2. Cunningham, J. J. \& Roussel, M. F. Cell Growth Differ. 12, 387-396 (2001).

3. Sherr, C. J. \& Roberts, J. M. Genes Dev. 9, 1149-1163 (1995).

4. Harper, J. W. Cancer Surv. 29, 91-107 (1997).

5. Sherr, C. J. \& Roberts, J. M. Genes Dev. 13, 1501-1512 (1999).

6. Cummingham et al. Mol. Cell. Neurosci. 19, 359-374 (2002).

7. Zindy, F. et al. Proc. Natl. Acad. Sci. USA 96, 13462-13467 (1999)

8. Chen, P. \& Segil, N. Development 126, 1581-1590 (1999).

9. Lowenheim, H. et al. Proc. Natl. Acad. Sci. USA 96, 4084-4088 (1999).

10. Zindy, F. et al. Mol. Cell. Biol. 20, 372-378 (2000).

11. Raphael, Y. British Med. Bulletin 63, 157-169 (2002)

12. Ryals, B. M. \& Rubel, E. W. Science 240, 1774-1776 (1988).

13. Thullberg, M., Bartek, J. \& Lukas, J. Oncogene 19, 2870-2876 (2000)

14. Zhang, P., et al. Genes Dev. 12, 3162-3167 (1998).

15. Zhang, P. et al. Genes Dev. 13, 213-224 (1999).

\title{
Small changes in Wnt signalling
}

Identifying components of the canonical Wnt signalling pathway has been at the forefront of developmental biology research since a link was made between Wnt, the mammalian homologue of Drosophila melanogaster Wingless (Wg), and the development of colon cancer. Although new members of the pathway have been cloned on a regular basis, most of these proteins have been agonists of signal transduction. In a recent issue of Nature, however, Randall Moon and colleagues describe the cloning of Chibby, a novel antagonist of the Wnt pathway that seems to function in the nucleus (Takemaru et al. Nature, 422, 905-909 2003).

Work from numerous labs has established the intricacies of the classical Wnt-Wg signalling pathway, in which the oncoprotein $\beta$-catenin/Armadillo(Arm) functions with the transcription factors TCF/Lef- 1 to positively transduce the signal to the nucleus. Inappropriate activation of the Wnt pathway results in increased gene expression of targets such as the cell cycle regulator CyclinD1, which are thought to be involved in the development of human colon cancer.

Takemura et al. identify Chibby as a novel mammalian protein that can interact with the carboxy-terminal transcription activation domain of $\beta$-catenin. Chibby (which means small in Japanese) is a novel protein with a nuclear localisation signal (NLS) and coiled-coil regions that seem to be conserved in flies. Consistent with the identification of a NLS, Chibby localization is primarily nuclear. Using the TCF/Lef-1 TOPFLASH reporter gene assay, the authors showed that overexpression of Chibby prevents cyclinD1 expression, whereas loss of Chibby function through morpholino experiments results in increased TOPFLASH activity. Therefore, it seems that Chibby could regulate the activation of $\beta$-catenin gene targets. Immunoprecipitation experiments indicate that Chibby can decrease binding between $\beta$-catenin and TCF/Lef1. Therefore, it is probable that Chibby antagonizes the signal transduction pathway by preventing the interaction of $\beta$-catenin and TCF/Lef- 1 .

As a result of sequence similarity between mammalian Chibby and the Drosophila homologue, the authors were keen to determine whether the action of Chibby was conserved between mammalian cell lines and the fruitfly. Drosophila embryos that
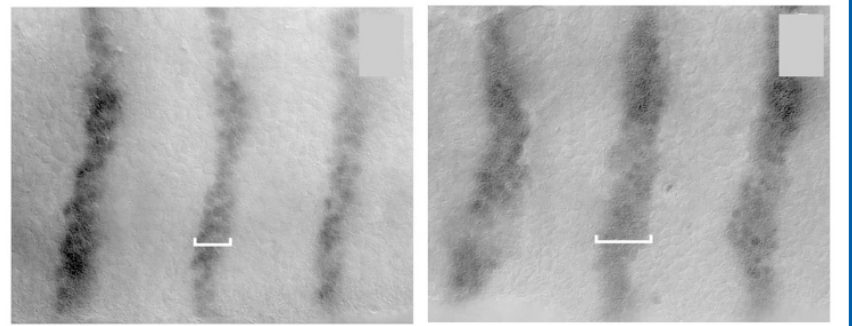

Ectopic activation of the Wg pathway in Chibby mutant flies. RNAi of chibby in Drosophila embryos results in expansion of the engrailed stripe in stage- 11 embryos. A wild-type embryo is shown on the left, a chibby mutant is shown on the right.

lack Chibby as a result of inhibitory RNA approaches have segment polarity phenotypes similar to those resulting from wingless overexpression in the embryo. These embryos also have increased expression of wingless-responsive genes in both the epithelium and the midgut. Genetic epistasis experiments in the midugt clearly indicate that Chibby functions upstream of Arm, demonstrating that not only is the sequence of Chibby conserved, but so is the function.

Yet again the research on Wnt-Wg signalling must be reassessed, now that another component of the transduction pathway has been identified. Chibby seems to be a nuclear protein with a proposed role in antagnoizing the pathway through inhibition of the $\beta$-catenin/Arm-TCF/Lef-1 transcription complex. How this new antagonist interacts with the other known transcriptional antagonists of Wg signalling, such as Groucho, is not yet clear. In addition, the possible relevance of this protein to the development of human cancer has yet to be established, although preliminary data show a reduction of Chibby in uterine and thyroid cancers. What is clear is that even although Chibby mutants themselves might be small, the advance on the Wnt research field could turn out to be large.

SARAH GREAVES 